\title{
A First Principles Investigation of Lithium Intercalation in $\mathrm{TiO}_{2}-\mathrm{B}$
}

Dwi Panduwinata and Julian D. Gale*

Nanochemistry Research Institute, Curtin University of Technology, GPO Box U 1987, Perth, 6845, Western Australia. Tel. +61 89266 3838, Fax. +61 892664699

Received Date ( inserted by publisher)

Revised Received Date (inserted by publisher)

\begin{abstract}
The intercalation of lithium into the polymorph of titania, $\mathrm{TiO}_{2}-\mathrm{B}$, has been examined using first principles methods, based on the Generalised Gradient Approximation within density functional theory. Three symmetry unique sites have been identified for the preferential location of lithium within the structure at low concentration, as well as the diffusion pathways between these sites. Lithium is found to bind most favourably at a site close to the titania octahedral layer, while the lowest activation energy for diffusion of $27 \mathrm{~kJ} / \mathrm{mol}$ is found for diffusion along the open channel parallel to the $b$ axis of the material. The need to activate lithium towards diffusion through the population of higher energy binding sites within the channel, along with the larger barrier for lithium to migrate through the sidewalls of nanotubular $\mathrm{TiO}_{2}-\mathrm{B}$, provides an explanation for many of the observed experimental electrochemical properties of this potential battery material.
\end{abstract}




\section{Introduction}

The current drive to lower carbon emissions has led to new vigour in the search for more efficient advanced materials for use in electrical storage. New forms of the titania polymorph $\mathrm{TiO}_{2}-\mathrm{B}$ have recently been synthesised by Armstrong et $a l^{[1,2]}$. in the form of nanotubes and nanowires. These structures can be produced in high yield and in relatively large quantities by an inexpensive and simple hydrothermal reaction between sodium hydroxide and anatase ${ }^{[1,2]}$. Combined with their high surface area morphologies, they have been shown to offer particular promise for utilisation as a negative electrode for rechargeable lithium ion batteries ${ }^{[1,3-6]}$.

$\mathrm{TiO}_{2}-\mathrm{B}$ is the least dense polymorph of $\mathrm{TiO}_{2}{ }^{[7-9]}$. In its nanotube form, $\mathrm{TiO}_{2}-\mathrm{B}$ has a greater

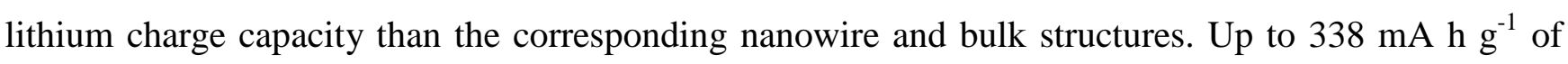
charge can be inserted into the nanotubes, leading to the final composition of $\mathrm{Li}_{1.01} \mathrm{TiO}_{2}-\mathrm{B}^{[5]}$. Lithium intercalation resulting in compositions of up to $\mathrm{Li}_{0.91} \mathrm{TiO}_{2}-\mathrm{B}\left(305 \mathrm{~mA} \mathrm{~h} \mathrm{~g}{ }^{-1}\right.$ ) can be obtained for the nanowire structure ${ }^{[6]}$. The lithium capacity of the nanotube exceeds the charge capacity of anatase and bulk $\mathrm{TiO}_{2}-\mathrm{B}$ structures, which have a maximum $\mathrm{Li}$ content of $\mathrm{Li}_{0.5} \mathrm{TiO}_{2}{ }^{[6]}$ and $\mathrm{Li}_{0.85} \mathrm{TiO}_{2}-\mathrm{B}^{[10]}$, respectively. The superior capacity for the nanotube structure can be attributed to the higher surface area, which provides greater electrode/electrolyte contact areas. In addition, there is more facile intercalation into nanotube structures due to the short diffusion pathways within the smaller dimension of the particles, which typically have a wall thickness of 25-30 $\AA^{[5]}$. Although there is some irreversibility on the first cycle, the charge/discharge cyclability of the nanotube/wire is excellent ${ }^{[1,3,5}$, 6]. The rate capability of the nanowire is superior to that of nanoparticulate anatase and $\mathrm{TiO}_{2}-\mathrm{B}$ with an average particle size comparable to the diameter of the wire ${ }^{[6]}$. This demonstrates the enhancement of the material properties due to its morphology, which renders this material particularly attractive for energy storage applications.

While experiment has provided considerable information regarding the overall characteristics of lithium intercalation within $\mathrm{TiO}_{2}-\mathrm{B}$, there has been little elucidated regarding the atomic details of the process. Here computational studies, especially those based on first principles quantum mechanics, are able to play a role, as they have done in the case of lithium incorporation into numerous other oxide materials ${ }^{[11-19]}$. They can give insight into the local bonding environment, energetics and diffusion behavior of the intercalated lithium within the host structure, which are often difficult to obtain unambiguously from experiments. In addition, it is also possible to predict properties, such as the intercalation voltages ${ }^{[12,17,20-22]}$ as a function of lithium content. 
To date, there has only been limited theoretical study of any kind of lithium intercalation behaviour into the $\mathrm{TiO}_{2}$-B structure. There has only been one prior published theoretical study of lithium intercalation in $\mathrm{TiO}_{2}-\mathrm{B},{ }^{[8]}$ which utilized the extended Huckel method ${ }^{[23]}$ mainly to investigate the bonding properties of the lithium intercalated bulk $\mathrm{TiO}_{2}-\mathrm{B}$ material based on the crystal orbital overlap population (COOP) formalism ${ }^{[24]}$. This method suggests that the $\mathrm{Li}$ ion prefers to intercalate at a site with five-fold oxygen coordination. It also predicts a small distortion of the original $\mathrm{TiO}_{2}-\mathrm{B}$ framework in order to achieve optimal bonding interactions. Due to the low screening of the $\mathrm{Li}$ ion repulsion, further filling of the $\mathrm{TiO}_{2}$ - $\mathrm{B}$ channels beyond a stoichiometry of $\mathrm{Li}_{0.5} \mathrm{TiO}_{2}$ became energetically unfavourable within this study.

In contrast to $\mathrm{TiO}_{2}-\mathrm{B}$, lithium intercalation in rutile and anatase has been studied extensively by Koudriachova et al. using density functional theory (DFT) calculations ${ }^{[20,22,25-27]}$. Full relaxation of structures for a wide range of insertion concentrations is used to identify the thermodynamically most stable configurations and the lithium intercalation sites within the rutile structure ${ }^{[20]}$. The rutile intercalation host is found to undergo significant deformation upon $\mathrm{Li}$ insertion, comensurate with the dense nature of the structure. Although lithium intercalation is thermodynamically favourable, the absence of lithium insertion into rutile at room temperature can be attributed to the kinetic restriction of the diffusion process which is ameliorated with increasing temperature ${ }^{[22]}$.

In this study, we present the results of a theoretical investigation of lithium ion intercalation into the bulk $\mathrm{TiO}_{2}$-B structure, based on density functional theory (DFT) calculations, in order to gain insight into the atomistic details of this process that may influence its performance as a prospective lithium ion battery electrode. Firstly, the stabilities of the various sites available for the lithium ions are examined at low concentration. Secondly, the mobility of lithium within the structure was probed by computing the activation barriers for lithium diffusion between the stable intercalation sites along the $a$, $b$ and $c$ lattice directions. Finally, the effects of lithium concentration on the voltage behaviour are investigated by constructing the average intercalation voltage curve. The results of this investigation provide new information regarding the properties of this lesser characterised polymorph and also offers explanations for some of the experimental characteristic properties ${ }^{[1,3,5,6]}$ of lithium incorporation into the different forms of $\mathrm{TiO}_{2}$-B structures, such as the nanotubes/wires.

\section{Methodology}


Lithium intercalation in $\mathrm{TiO}_{2}-\mathrm{B}$ has been investigated using density functional theory as implemented within the SIESTA methodology and program ${ }^{[28]}$. Here the core electrons and nuclei are represented through non-local norm-conserving pseudopotentials of the Troullier-Martins form ${ }^{[29]}$, while the valence Kohn-Sham eigenstates are expanded as a linear combination of pseudo-atomic orbitals (PAOs). The PAOs are constructed from the numerical solution to the pseudised atomic problem, subject to a confining potential that ensures that the orbitals are strictly local in order to ensure linear-scaling construction of the Kohn-Sham Hamiltonian and overlap matrix. In the present work, an energy shift associated with the orbital confinement of 0.01 Ry was chosen. In order to ensure smoothness of the orbital derivatives at the cutoff, the soft-confinement scheme of Junquera et al. ${ }^{\text {[30] }}$ was employed with a potential of $50 \mathrm{Ry}$ and the radius at which the asymptotically infinite potential begins to be applied was set to 0.8 of the cutoff radius. The basis set was decontracted using the previously proposed split-norm scheme with an outer norm fraction of 0.3 to generate a basis set of double-zeta polarised (DZP) quality for all atoms except titanium, where orbitals of $\mathrm{f}$ angular momentum were not included. In order to obtain a reliable description of titanium, the 3p semi-core states were explicitly included in the valence, while the overlap with the 3 s levels is described through non-linear core corrections ${ }^{[31]}$. This treatment is found to be important in order to reproduce the expected trend of $\mathrm{TiO}_{2}$ phase stability and in correctly describing lithium intercalation into the rutile structure $^{[20,25,27,28,32-40]}$.

All calculations have been performed using the Generalized Gradient Approximation (GGA) with the functional due to Perdew, Burke and Ernzerhof (PBE). ${ }^{[41]}$ While Zhou et al ${ }^{[42]}$ have demonstrated that the use of GGA+U leads to approximately an order of magnitude improvement in quantitative prediction of cell voltages, through the improved description of the energy of transition metal $d$-electron states by reduction of the self-interaction error, the qualitative trends obtained in the absence of an on-site Hubbard U parameter are typically correct. Furthermore, the effective U values (U-J) used in previous LDA+U studies involving titanium are much smaller than those employed for later transition metals. ${ }^{[43]}$ Consequently, we have chosen to employ just the pure GGA for the present study.

Spin-polarization was included under the constraint of fixed total spin in order to describe the intercalation of lithium with an odd number of electrons. The Kohn-Sham eigenstates were solved for through matrix diagonalization with sampling of the Brillouin zone on a uniform Monkhorst-Pack grid of k-points. The dimensions of the sampling mesh were determined according to the method of Moreno \& Soler ${ }^{[44]}$, with an equivalent real space cutoff of $13 \AA$. 
For the evaluation of the Hartree and exchange-correlation contributions, the electron density was expand in an auxiliary basis set consisting of a uniform grid of points in real space with an effective kinetic energy cutoff of 400 Ry. To try to maximise translational invariance, the grid cell sampling scheme was employed based on two points at displacements of $(0,0,0)$ and $(1 / 2,1 / 2,1 / 2)$ as fractions of the mesh spacing. The numerical parameters associated with both real and reciprocal space meshes were chosen to ensure convergence of the total energy of rutile to within $0.01 \mathrm{eV}$.

All geometry optimisations were performed using conjugate gradients with a convergence criteria of $0.01 \mathrm{eV} / \AA$ for the atomic forces and a pressure tolerance of 200 bar with respect to the unit cell. A graphical user interface, GDIS ${ }^{[45]}$ was used to build the SIESTA input files and to visualise the output of the calculations.

Determination of lithium intercalation sites. In order to determine lithium intercalation sites within the bulk $\mathrm{TiO}_{2}-\mathrm{B}$ structure, a stoichiometry of $\mathrm{Li}_{1 / 8} \mathrm{TiO}_{2}-\mathrm{B}$ was initially considered. In order to predict the possible intercalation sites, the following considerations were taken into account. Firstly, the inserted $\mathrm{Li}$ atom is ionised, donating charge to the lattice ${ }^{[8,20,26]}$. The position of $\mathrm{Li}$ ion in the $\mathrm{TiO}_{2}-\mathrm{B}$ structure is largely influenced by its interaction with the nearby oxygen atoms. It is expected that the most favourable configuration corresponds to the geometry with the highest degree of oxygen coordination $^{[8]}$. Secondly, a stable intercalation should produce effective screening of the electrostatic repulsion due to $\mathrm{Li}-\mathrm{Li}$ and $\mathrm{Li}-\mathrm{Ti}$ ions interactions ${ }^{[8,20,26,27]}$. The effective screening can be achieved by increasing Li-Ti distances and the degree of oxygen coordination ${ }^{[20]}$. In addition, it seems likely that the number of different low-energy positions available for intercalation is determined by the relative size of the Li guest ion and the space available within the lattice.

The Li ion was initially inserted into nine different possible intercalation sites within the asymmetric unit of the bulk structure for the $\mathrm{Li}_{1 / 8} \mathrm{TiO}_{2}-\mathrm{B}$ stoichiometry. The system was fully optimised in each case to achieve a local minimum in the total energy, which corresponds to a possible intercalation site. The stable sites for lithium intercalation into the bulk $\mathrm{TiO}_{2}-\mathrm{B}$ structure towards the dilute limit of concentration $\left(\mathrm{Li}_{1 / 48} \mathrm{TiO}_{2}-\mathrm{B}\right)$ were also investigated in order to determine the intrinsic structural preferences without the complexity of lithium-lithium interactions. To achieve this, the Li ion is inserted into a supercell structure made up of $1 \times 3 \times 2$ unit cells, where the ratio of the dimensions were chosen in order to try to achieve close to uniform spacing of lithium in all crystallographic directions. The energy required for lithium intercalation is then computed based on the assumption that Li atoms are extracted from bulk lithium metal. 
Lithium diffusion. Although in principle the occupancy of the various lithium intercalation sites can be predicted from thermodynamic considerations, it is also important to examine the kinetics of diffusion since this could potentially prevent sites from being accessed. The mobility of the Li ions is examined by computing the constant volume energy surfaces for a single lithium diffusing in a material of $\mathrm{Li}_{1 / 48} \mathrm{TiO}_{2}-\mathrm{B}$ stoichiometry. Lithium is assumed to move through the $\mathrm{TiO}_{2}-\mathrm{B}$ structure by 'hopping ${ }^{[8]}$ from occupied to unoccupied intercalation sites. Lithium diffusion between intercalation sites along the $a, b$ and $c$ directions is considered. At each position the energy is evaluated after geometry relaxation of the internal coordinates. Consideration of the lithium-lithium interactions and their influence on diffusion as a function of loading would lead to more pathways than it is currently feasible to examine based on first principles techniques. However, it is likely that such effects are going to be secondary to the intrinsic preferences for occupied-unoccupied site hops, especially since the reduced band gap with increased lithium content will lead to improved dielectric screening.

Constrained optimisations are employed to determine the total energy for each configuration along a pathway. In this case, the motion of the $\mathrm{Li}$ ion and four $\mathrm{Ti}$ atoms are constrained along the diffusion direction, while directions normal to this direction are allowed to fully relax for all atoms. Constraints are applied to the $\mathrm{Ti}$ atoms that are located at large distances from the $\mathrm{Li}$ ion in order to break translational invariance. The geometric constraints are implemented using the Z-matrix facility within SIESTA ${ }^{[46]}$. The relative energy surfaces are then constructed with respect to the minimum energy configuration.

Lithium concentration. In order to examine the effect of lithium concentration, an average intercalation voltage is computed for lithium incorporation into the bulk $\mathrm{TiO}_{2}$-B (cathode) at different concentrations. The $\mathrm{Li}_{\mathrm{x}} \mathrm{TiO}_{2}-\mathrm{B}$ ordering configuration is considered for $\mathrm{x}=0,1 / 48,1 / 24,1 / 16,1 / 8$ and 1/2. In this case, lithium ions are assumed to be generated from bulk lithium metal anode. The average intercalation voltage is computed using the method described by Aydinol et al. ${ }^{[32]}$. This method allows one to compute the average voltage between two intercalation limits, based on the approximation that the entropic contribution to the free energy is negligible. The calculation requires the values of the total energies for bulk lithium metal and $\mathrm{Li}_{\mathrm{x}} \mathrm{TiO}_{2}-\mathrm{B}$ as a function of composition. The voltage at a particular composition is then determined by finite differencing the total energies for the configurations either side of that particular value of lithium content.

The most stable configuration is determined by initially considering the possible permutations for lithium ordering at each concentration while allowing for space-group symmetry to focus on unique 
configurations. Candidate low energy configurations were created based on the following considerations. Firstly, the Li ions will tend to populate the intrinsically most stable sites first, as determined for the low concentration limit. Secondly, the $\mathrm{Li}$ ions will tend to maximise the $\mathrm{Li}-\mathrm{Li}$ separation in order to reduce the ionic repulsion ${ }^{[8]}$.

At least five suspected low energy initial configurations were considered for each stoichiometry. An energy calculation is then performed for each of the candidate configurations at fixed atomic coordinates and unit cell parameters. The computed energy of the system should indicate the approximate structural stability of the initial configuration. The lowest energy structure is chosen and further optimised until the full relaxation of the internal atomic coordinates and the cell parameters is achieved. Although it cannot be guaranteed, these configurations should be close to the minimum energy structure for each stoichiometry. In order to be certain a more extensive study of any potential disorder would be required by combining the first principles results with interatomic potentials and cluster expansion methods, as applied to disordered materials where configurational entropy becomes important $^{[47]}$.

\section{Results and Discussion}

\section{Bulk $\mathrm{TiO}_{2}-\mathrm{B}$}

Prior to the investigation of lithium ion intercalation, it is important to verify how well the general simulation conditions reproduce the bulk properties of $\mathrm{TiO}_{2}-\mathrm{B}$. In this case we consider the reproduction of experimental lattice parameters and the trend in the relative phase stability of $\mathrm{TiO}_{2}-\mathrm{B}$ with respect to rutile and anatase.

The bulk structure of $\mathrm{TiO}_{2}-\mathrm{B}$ was first synthesised by Marchand et al. in $1980^{[10]}$ by ionexchanged of $\mathrm{K}^{+}$for $\mathrm{H}^{+}$in $\mathrm{K}_{2} \mathrm{Ti}_{4} \mathrm{O}_{9}$ to form a hydrated hydrogen titanate. It was then transformed to $\mathrm{TiO}_{2}-\mathrm{B}$ by heating at $500^{\circ} \mathrm{C}^{[7,9,10]}$. $\mathrm{TiO}_{2}-\mathrm{B}$ has a monoclinic structure with experimental lattice parameters of $a=12.1787(10) \AA, b=3.7412(2) \AA, c=6.5249(5) \AA$ and $\beta=107.054(4)^{\circ}[7,9]$. The framework of the $\mathrm{TiO}_{2}-\mathrm{B}$ structure is constructed from corrugated sheets of edge- and corner-sharing $\mathrm{TiO}_{2}$ octahedra that are linked by bridging oxygen atoms to form a three-dimensional network ${ }^{[7,9]}$ (Figure 1). Consequently, this structure is more open than rutile, anatase or brookite, with infinite channels that are perpendicular to the (010) face. This structural feature is a major factor in making this material an effective host for lithium ion intercalation. Therefore, it is important that the computational 
method and conditions used reproduce the structure accurately and that any structural deformations induced by lithium intercalation can be simulated correctly.

Starting from the experimental values of lattice parameters of bulk $\mathrm{TiO}_{2}-\mathrm{B}$, the structure is optimised until full relaxation of the internal coordinates and the cell parameters is achieved according to the tolerances previously specified. The resulting calculated lattice parameters are $a=12.3512 \AA, b=$ $3.7904 \AA, c=6.6015 \AA$ and $\beta=107.0684^{\circ}$. They are in agreement with the experimental values ${ }^{[7,9]}$ to within 1.42\%. The magnitude of this error is consistent with the expectation that the use of the PBE functional, along with most other GGAs, tends to overestimate the computed cell parameters by $1 \%{ }^{[35]}$. The slightly larger error than this target is primarily due to the incompleteness of the basis set with respect to the planewave limit.

The semicore pseudopotential ${ }^{[31]}$ treatment on titanium is crucial in order to produce the expected trend of $\mathrm{TiO}_{2}$ phase stability where rutile is the most stable structure followed by anatase and $\mathrm{TiO}_{2}-\mathrm{B}$, respectively. The use of the DZP basis set produces the expected trend of phase stability as seen experimentally. In this case $\mathrm{TiO}_{2}$ - $\mathrm{B}$ is less stable by $2.4 \mathrm{~kJ} / \mathrm{mol}$ with respect to rutile. However, it should be noted that when higher quality basis sets are utilized, anatase is predicted to be more stable than rutile, in line with the majority of planewave studies and thus the correct ordering is fortuitous. In the case of a triple-zeta double-polarised basis set, anatase is more stable by $0.29 \mathrm{~kJ} / \mathrm{mol}$ with respect to rutile. Similar difficulties with respect to the rutile-anatase energy difference were also reported in the

previous localised orbital study by Muscat et $a l^{[35]}$ where the the computed properties of the bulk $\mathrm{TiO}_{2}$ phase are sensitive to the treatments of the exchange and correlation, as well as the numerical approximations. For the present work, the DZP quality of basis set is found to be adequate since the energies of different sites within the same $\mathrm{TiO}_{2}-\mathrm{B}$ phase are important rather than the relative stability of alternative phases.

\section{Lithium ion intercalation sites}

Having explored a range of lithium initial positions for the $\mathrm{Li}_{1 / 48} \mathrm{TiO}_{2}-\mathrm{B}$ stoichiometry, three distinct stable lithium intercalation sites were identified, as shown in Figure 2. Here the absolute energy of lithium incorporation is computed relative to bulk $\mathrm{TiO}_{2}-\mathrm{B}$ and the assumption that lithium ion was extracted from lithium metal. 
As can be seen from Table 1, the computed energies indicate preferential binding in the pentacoordinated site shown in Figure $2 \mathrm{C}$, located at fractional coordinates of $(0.68,0.66,0.50)$. At this site $\mathrm{Li}$ ion achieves the most stable five-fold coordination by oxygen atoms. Li ion insertion into this site at the dilute limit of concentration causes reduction of the lattice symmetry from monoclinic (C $12 / \mathrm{m} 1)$ to Triclinic (P 1) and a small expansion of the $b$-lattice constant by $0.23 \%$. This leads to an overall volume increase of $0.36 \%$.

The second most stable site is located at a distorted octahedral site, shown in Figure 2B, at fractional coordinates of $(0.50,0.63,0.50)$. In this environment, $\mathrm{Li}$ ion effectively has two-fold coordination by the oxygen atoms that bridge the layers in the structure, with four more distant oxygens also weakly coordinating it. The Li ion is located slightly off-centre from the high symmetry point within the open channel of $\mathrm{TiO}_{2}$-B. Insertion at the dilute limit into site $\mathrm{B}$ causes a smaller, when compared to site C, overall expansion of the unit cell volume by $0.13 \%$. This is to be expected since site B is situated at the most open part of the channel within the material and so little distortion of the structure is required to accommodate a small cation.

The least stable lithium intercalation occurs at the second penta-coordinated site, shown in Figure 2A, located at fractional coordinates of $(0.56,0.66,0.70)$. The oxygen atoms form a triangular bipyramidal structure, where $\mathrm{Li}$ ion effectively experiences three-fold coordination with the nearest oxygen atoms. Insertion of lithium at the present concentration at site A again causes a small expansion of the overall cell volume by $0.33 \%$, very similar to site C.

The favourability of intercalation into a particular site seems to be influenced by the interplay of two different factors. On the one hand, lithium would ideally favour maximising the coordination number with oxygen, while reducing the Li-Ti repulsion. However, distorted five-fold coordination is found in both the most and least stable sites. Hence, this has to be balanced against the lattice strain induced by the presence of the cation, which favours sites $\mathrm{B}$ and $\mathrm{C}$ that both reside within the more open channel region. It is the combination of these two factors that makes site $\mathrm{C}$ the preferred one, though the energy difference to site B is close to the limit of the accuracy of current non-local density functionals. Overall, it appears that the steric argument is the dominant factor of the two highlighted.

Li ion intercalation in the $\mathrm{TiO}_{2}-\mathrm{B}$ structure was previously studied by Nuspl et al. ${ }^{[8]}$ based on extended Huckel theory ${ }^{[23]}$. This earlier work also suggested that the Li ion prefers to adopt five-fold coordination, in agreement with the present study, except that the penta-coordinated site A, shown in 
Figure 2A, was proposed as the minimum energy structure. However, the structural stability was derived for a stoichiometry of $\mathrm{Li}_{1 / 2} \mathrm{TiO}_{2}-\mathrm{B}$ and assumed an ideal geometry for the host lattice ${ }^{[8]}$. Given these two differences, some quantitative discrepancy is to be expected, especially since the present work indicates that the local oxygen environment undergoes some distortion on lithium incorporation, thus limiting the validity of the second approximation made in the previous work. In addition, Nuspl et al. did not consider Li ion intercalation at the most favourable penta-coordinated site (shown in Figure 2C) and the distorted octahedral site B.

Previous density functional theory studies of Li ion intercalation into the rutile and anatase phases of $\mathrm{TiO}_{2}$ have been presented by Koudriachova et al. ${ }^{\left[20,{ }^{22]}\right.}$. Contrary to lithium intercalation in the $\mathrm{TiO}_{2}-\mathrm{B}$ structure, significant distortions of the original structures were observed for intercalation in rutile and anatase. In the case of anatase, initial intercalation proceeds as a single reaction at low concentration (for $\mathrm{Li}_{0.05}-0.1 \mathrm{TiO}_{2}$ ) and further insertion produces a two phase equilibrium of Li-poor and Li-rich regions ${ }^{[22]}$. This was explained in terms of strong local distortions of the structure due to the localisation of the donated charge on the $\mathrm{Ti}$ ion during the phase transformation ${ }^{[26]}$. Lithium intercalation into the octahedral site is strongly favourable in the case of rutile. Similar to the intercalation in anatase, this site accommodates the $\mathrm{Li}$ ion with significant structural deformation involving anisotropic expansion of the lattice parameters ${ }^{[20]}$. $\mathrm{TiO}_{2}$ - $\mathrm{B}$ seems to provide a more facile structural framework for Li ion intercalation since small structural changes are observed upon lithium insertion. $\mathrm{TiO}_{2}$-B has a lower structural density than rutile or anatase, and it forms a one dimensional channel along the $b$ direction that would allow Li ion to diffuse through the structure to reach the stable penta-coordinated intercalation site without producing significant structural deformation.

\section{Lithium diffusion}

Although lithium ion intercalation into the $\mathrm{TiO}_{2}-\mathrm{B}$ structure has been observed experimentally $^{[5,6,10,33,40,48]}$ and confirmed to be thermodynamically favoured in the present work, the accessibility of the stable intercalation sites ultimately depends on the kinetics of the diffusion process. In order to examine Li ion mobility, constant volume energy surfaces for a single Li diffusion have been computed in a supercell structure of $\mathrm{Li}_{1 / 48} \mathrm{TiO}_{2}-\mathrm{B}$ stoichiometry. The diffusion pathways examined between sites are illustrated schematically in Figures 3a-c, with the corresponding energy 
profiles shown in Figures 4-6. Three overall diffusion pathways have been considered corresponding to lithium migration parallel to each of the crystallographic axes.

In order to determine the energy surface for diffusion, the lithium coordinate was fixed at a given value in the appropriate direction and the remaining degrees of freedom allowed to relax, along with those of the surrounding titania structure. The coordinates of a further set of $\mathrm{Ti}$ atoms in a single plane, remote to the lithium, were also fixed in order to prevent overall translation of the lattice. Constrained diffusion calculation under constant volume conditions were chosen because $\mathrm{Li}$ ion diffusion at the dilute limit of concentration is expected to yield only a small change in the lattice parameters for lithium insertion at this concentration, as previously demonstrated for sites A, B and C. Furthermore, in the real situation where a stoichiometric number of lithium ions do not diffuse concurrently, the overall bulk unit cell will not be able to respond to individual ion movements. The optimisations of the energy minima demonstrate that the internal relaxation of the coordinates is the predominant change in response to lithium ion location.

Lithium migrates through the $\mathrm{TiO}_{2}-\mathrm{B}$ structure by 'hopping ${ }^{\text {,[8] }}$ from occupied to unoccupied intercalation sites. In order to examine $\mathrm{Li}$ ion mobility along the $a$ direction, the diffusion pathway is divided into two segments ${ }^{[8]}$. The first segment corresponds to the migration pathway of lithium from the first C intercalation site (indicated by C1 in Figure 3a) located at the fractional coordinates (0.68, $0.66,0.50)$ to the $\mathrm{B}$ site located at $(0.50,0.63,0.50)$. The energies were computed for different positions of $\mathrm{Li}$ ion along the pathway for $0.51 a<x<0.68 a$. The energy barrier is computed to be 12.2 $\mathrm{kJ} / \mathrm{mol}$ and occurs for a lithium coordinate of $0.56 a$. As lithium diffuses along this pathway $(\mathrm{C} 1 \rightarrow \mathrm{B})$, it moves through the small oxygen gap of $3.62 \AA$ corresponding to a distance between oxygens number 4 and 5, as labelled in Figure 2C.

The second segment of this pathway corresponds to the migration pathway of lithium from the first $C$ intercalation site located at approximate fractional coordinates of $(0.68,0.66,0.50)$ to the next equivalent site by structural symmetry, indicated as C2 in Figure 3a, located at $(0.78,0.54,0.50)$. The energies were computed as a function of lithium position along the pathway for $0.66 b>y>0.57 b$ deduced from the symmetry of the structure. The energy barrier for this pathway is $96.9 \mathrm{~kJ} / \mathrm{mol}$, which occurs when lithium has a $y$ coordinate of $0.58 b$. In this case, the overall energy barrier for diffusion along the $a$ direction corresponds to the pathway between the $\mathrm{C}$ intercalation sites. As shown in Figure $4 \mathrm{~b}$, the energy profile for diffusion between the two symmetry related C sites is not symmetric as a result of the strong distortion of the structure during migration. Hence, in order to validate the transition 
state with respect to numerical factors the barrier was computed in both directions. Figure $4 \mathrm{~b}$ demonstrates that the two potential energy surfaces are mirror images of each other about the centre of symmetry between the two C sites and therefore are in excellent agreement.

Only one important diffusion pathway is considered for lithium diffusion along the $b$ direction. This pathway corresponds to the migration of Li ion from the first B intercalation site (B1 in Figure 3b) located at $(0.50,0.63,0.50)$ to the next equivalent intercalation site by symmetry located at $(0.50,0.37$, 0.50). The energies were computed for different positions of $\mathrm{Li}$ ion along the pathway in the range $0.63 b<y<0.49 b$ derived from the structural symmetry. The overall energy barrier for this pathway, shown in Figure 5, is $27.0 \mathrm{~kJ} / \mathrm{mol}$ located for lithium sited at $0.50 \mathrm{~b}$, which represents the mid point between the two B sites.

In order to examine lithium mobility along the $c$ direction, the diffusion pathway is again divided into two segments. The first segment corresponds to the migration pathway between the B intercalation site located at the fractional coordinates $(0.50,0.63,0.50)$ and the first A intercalation site (indicated by A1 in Figure 3c) located at $(0.56,0.66,0.70)$. The energies were computed as a function of lithium position along the pathway for $0.50 c<z<0.68 c$, as shown in Figure 6a. The computed energy barrier is $37.6 \mathrm{~kJ} / \mathrm{mol}$, which occurs for a lithium coordinate of $0.64 \mathrm{c}$. The second segment corresponds to the migration pathway between the first A intercalation site located at $(0.56,0.66,0.70)$ and the next equivalent intercalation site by symmetry (indicated by A2 in Figure 3c) located at (0.36, 0.66, 0.80). The energies were computed as a function of lithium position along the pathway for $0.70 \mathrm{c}$ $<z<0.77 c$, illustrated in Figure 6b, based on structural symmetry. The energy barrier is $36.4 \mathrm{~kJ} / \mathrm{mol}$ located at $0.76 c$. The overall energy barrier for diffusion along the $c$ direction corresponds to the pathway between A1 and A2 intercalation sites.

As summarised in Table 2, lithium mobility is expected to favour the directions in the order of $b$ $>c>>a$ based on the overall activation energy barrier heights. The $\mathrm{TiO}_{2}-\mathrm{B}$ structure forms open channels parallel to the $b$-axis, which are sufficiently large enough to be able to accommodate lithium diffusion without inducing appreciable deformation. In contrast, diffusion in any other crystallographic direction involves the cation migrating through small rings of cross-linked octahedra and thus induces appreciable localised strain. This causes the strong asymmetry in the energy barriers for lithium diffusion across $\mathrm{C} 1 \leftrightarrow \mathrm{C} 2$ and $\mathrm{A} 1 \leftrightarrow \mathrm{A} 2$ pathways shown in Figures 4b and 6b, respectively. This nonsymmetrical behaviour is similar to stick-slip behaviour, where the ion migration induces distortion of oxygen environment until a threshold is reached at which the lithium can pass through the face of the 
coordination polyhedron. Although we have only considered the activation energy and not the attempt frequency prefactor to the rate constants, it is unlikely that this would significantly alter the present findings since the vibration of the lithium is unlikely to show strong anisotropy and variation between sites.

Diffusion along the $c$ direction is expected to follow a 'zigzag' path due to the offset of the symmetry related A sites parallel to the $a$-axis on opposite sides of the titania layer, as shown in Figure 3c. Migration involves a three step process involving an activated hop from the B site in the channel to the A site on one side of the octahedral layer, followed by a transition state leading to an A site on the opposite side of the layer. The final step, from the second A-site back to a B-site, has a relatively low barrier of $14.2 \mathrm{~kJ} / \mathrm{mol}$, being driven by the lowering of energy associated with the transition between the minima, and so is not expected to significantly influence the rate of diffusion. The overall barrier height to diffusion for this direction is $37.6 \mathrm{~kJ} / \mathrm{mol}$ corresponding to the diffusion from the $\mathrm{B}$ site to the A site.

The highest energy barrier of all corresponds to lithium diffusion parallel to the $a$ direction. Here there is a low energy barrier for migration of lithium from the B site in the channel to the more stable $\mathrm{C}$ site, followed by a more significant activation energy to attain the adjacent $\mathrm{C}$ site. This is perhaps the most surprising result, in that at first sight the pathway between the $\mathrm{C}$ sites appears relatively open and so facile diffusion is expected. However, the reason that the $\mathrm{C}$ site is the most stable binding position is because the structure is able to distort to maximise the local coordination of lithium; an effect that has to be reversed to facilitate diffusion. Based on the result that diffusion should occur most readily along the $b$-direction via hopping between $\mathrm{B}$ sites, the low activation energy for lithium to leave the channel and attain the more stable $\mathrm{C}$ sites represents a trapping mechanism that will slow diffusion.

Experimentally, the growth of microfibrous $\mathrm{TiO}_{2}$-B tends to be predominantly parallel to the $b$ axis ${ }^{[1,39]}$. This directional growth behaviour also occurs in the case of $\mathrm{TiO}_{2}-\mathrm{B}$ nanotube structures. High-resolution transmission electron microscope analysis indicates that the crystal structure orients in the nanotube structure in such a way that the $b$-axis lies along the longitudinal axis of the tube and the $a$-axis is parallel to the radial vector for the tube ${ }^{[5]}$. Thus, the rate determining step for diffusion in these structures should correspond to lithium transfer across the wall of the nanotube and surfaces of the nanowire. 
Cyclic voltammogram measurements obtained for various $\mathrm{TiO}_{2}-\mathrm{B}$ structures (bulk, nanowire and nanotube) revealed the presence of S-peaks with small peak-to-peak splittings, which are believed to be the signature of the $\mathrm{TiO}_{2}-\mathrm{B}$ behaviour ${ }^{[3,5,48]}$. The existence of S-peaks was interpreted as Faradaic pseuodocapacitive Li-storage behaviour due to the surface confinement during the Li-transfer process $^{[47]}$. This process was initially rationalised based on the idea of fast lithium transfer through the parallel channels of the $\mathrm{TiO}_{2}-\mathrm{B}$ lattice ${ }^{[8,48]}$. These channels are abundant at the cross section of the microfibrous particle and run perpendicular to the (010) face of $\mathrm{TiO}_{2}-\mathrm{B}$. During the lithium insertion, Li ions interact with the overall surface of the particle and could potentially penetrate into the bulk fibre through the radial direction. Assuming the channels transfer Li ions rapidly within the particle, the rate determining process is attributed to the transfer across the surfaces, which may provide the mechanism for surface confinement.

The computed diffusion energy barriers presented in Table 2 confirm the high lithium mobility through the channels and that the overall kinetics of the intercalation process should be determined by lithium migration across the surface, which corresponds to more highly activated migration pathways through the structure. Therefore, the high diffusion energy barrier across the surfaces is indeed likely to be responsible for the formation of the S-peaks. The fact that these peaks are present in the different types of $\mathrm{TiO}_{2}-\mathrm{B}$ structures ${ }^{[3,5,48]}$ indicates an inherent characteristic diffusion behaviour of the bulk $\mathrm{TiO}_{2}-\mathrm{B}$ structure.

The experimental obsevation of pseudocapacitive lithium storage behaviour ${ }^{[48]}$ can be attributed to the existence of a reversible intercalation site in the vicinity of the channels in $\mathrm{TiO}_{2}-\mathrm{B}$ structure. This site corresponds to the three-fold pentacoordinated site shown in Figure 2A. The diffusion energy barrier for intercalation at this site ( $\mathrm{B} \rightarrow \mathrm{A} 1$ pathway) is higher than the one along the $b$ direction. Lithium could temporarily undergo stable intercalation at the (001) face and produce the pseudocapacitive characteristic. This process is expected to be reversible since lithium prefers to migrate back towards the octahedral site and diffuse through the channel.

Observation of pseudocapacitive lithium storage in other structures with one-dimensional channels, such as ramsdellite ${ }^{[49]}$ and hollandite ${ }^{[36]}$, has not been confirmed. The hollandite structure shows poor lithium storage electrochemistry despite the fact that it exhibits large open channels in the lattice ${ }^{[36]}$. Coincidently, good pseudocapacitive performance in $\mathrm{TiO}_{2}-\mathrm{B}$ corresponds to structures with open pathways for Li-transport and proper reversible intercalation site located in close vicinity of these pathways $^{[48]}$. 
The kinetics of the $\mathrm{Li}$ ion transfer into the $\mathrm{TiO}_{2}-\mathrm{B}$ nanostructures is dominated by migration across the (100) and (001) faces. The contribution of the (010) face can be neglected since it engages in $\mathrm{Li}$ ion transfer occurring just at the tip of the fibre or the tube. There is a significant difference in the effective energy barrier for diffusion through the (100) and (001) faces, which could provide a reason for the presence of the peak-to-peak splitting of the S-peaks during the lithium insertion process. This behaviour is similar to the lithium insertion anisotropy in the anatase phase ${ }^{[50]}$. Thus the characteristic behaviour of the bulk crystal structure is maintained throughout the different types of $\mathrm{TiO}_{2}-\mathrm{B}$ structures.

The fine structure of the S-peaks changes as the morphology of $\mathrm{TiO}_{2}$ - $\mathrm{B}$ particle is transformed from the bulk structure (typical diameter of 100-200nm and length $\sim 1-2 \mu \mathrm{m}$ ) to nanowires (with diameters of $20-40 \mathrm{~nm}$ and length up to several micrometers $)^{[3,5,6]}$. In the case of the Li-insertion process, the peak-to-peak features become less well resolved. The S-peak 'smoothing ${ }^{\text {,[5] }}$ can be attributed to the possible changes in the lattice structure due to the presence of lattice strain from the formation of the cylindrical structures. The $\mathrm{TiO}_{2}-\mathrm{B}$ structure will be highly strained as it forms the wall of the nanotube structure. The small particle dimension and the curvature of the nanotube walls or the nanowire surface can potentially alter the energetics of the lithium intercalation. Since the $a$-axis of the $\mathrm{TiO}_{2}-\mathrm{B}$ lies parallel to the radial direction of the tube, the curvature of the tube may produce expansion of the (100) and (001) faces, which alters the energy barrier for diffusion along the $a$ and $c$ directions. Changes in the fine structure of the S-peaks during the discharge process can be attributed to the alteration of the energetics of lithium diffusion at high concentration.

Initial irreversible capacity loss is a common phenomenon that happens for all $\mathrm{TiO}_{2}-\mathrm{B}$ structures. Lithium intercalation in the $\mathrm{TiO}_{2}-\mathrm{B}$ nanowires leads to $3 \%$ of discharge capacity loss ${ }^{[3]}$. On the contrary, there is significant irreversibility for intercalation in the $\mathrm{TiO}_{2}-\mathrm{B}$ nanotube structure indicated by $29 \%$ of discharge capacity $\operatorname{loss}^{[5]}$. Irreversible capacity loss was also observed for intercalation into bulk $\mathrm{TiO}_{2}-\mathrm{B}^{[3,5]}$. It seems that the magnitude of irreversible discharged capacity increases as a function of increasing surface area. The possible formation of a Solid Electrolyte Interface (SEI) layer, commonly occurs as a problem on graphite electrodes, was dismissed as a possible cause for the irreversible capacity loss in $\mathrm{TiO}_{2}-\mathrm{B}$ structures ${ }^{[3,6]}$. Transmission electron micrographs and AC impedance plot analysis reveal no evidence of SEI layer formation at these surfaces. 
Armstrong et al. ${ }^{[1,3,5,6]}$ suggested a few possible reasons for the initial irreversible capacity loss. Firstly, during the intercalation process, the Li ion could become trapped in certain sites due to the minor relaxation of the structure. This makes it more difficult for lithium to be extracted during the following charge/discharge cycles. From the results of the present DFT calculations, a lithium 'trapping' mechanism can be postulated based on competition between the high mobility along the channel and the favourable lithium diffusion from the off-centred octahedral site to the most stable pentacoordinated site (indicated by B $\rightarrow \mathrm{C} 1$ in Figure 3a). The C1 intercalation site is easily accessible due to the low energy barrier for $\mathrm{B} \rightarrow \mathrm{C} 1$ pathway . Thus, lithium could become trapped at this more stable site once the electrochemical potential is removed that favours channel diffusion. Regaining the mobility of these lithium ions then requires reactivation of the system through driving the ions back to the $\mathrm{B}$ sites.

The second possible reason for irreversible capacity loss is the poor electronic conductivity at the surface regions, which may lead to the formation of an insulating layer within the $\mathrm{TiO}_{2}-\mathrm{B}$ near the surface, and therefore provide significant kinetic hindrance during lithium extraction ${ }^{[3,5,6]}$. Lithium ions could potentially be trapped in the C1 intercalation site and induce the formation of an insulating layer. This layer may impede lithium migration across the (100) face during their extraction. The current DFT investigation of lithium intercalation at this site does not indicate significant lattice deformation that would potentially be sufficient to lead to the formation of a new phase or superstructure near the surface. This is in agreement with the experimental observation based on the Xray measurements ${ }^{[1,3,5,6]}$. In addition, NMR investigations indicate that all occupied intercalation sites are equivalent for the $\mathrm{Li}_{1 / 2} \mathrm{TiO}_{2}-\mathrm{B}$ stoichiometry ${ }^{[10,33,40]}$ with no indication of the formation of new phases. Thus both theory and experiment indicate that the capacitive loss is likely to be related to the subtle changes in the $\mathrm{TiO}_{2}$-B structure after lithium intercalation. However, further work is necessary in order to investigate the possible surface contribution to the initial lost of discharge capacity ${ }^{[3,5]}$. From the computer modelling point of view, lithium ordering for interfacial intercalation and the diffusion behaviours across the surfaces must be investigated to further understand the surface contribution on the lithium storage capacity in $\mathrm{TiO}_{2}-\mathrm{B}$.

\section{Lithium concentration.}


The effect of lithium concentration has been investigated by exploring a range of compositions for $\mathrm{Li}_{\mathrm{x}} \mathrm{TiO}_{2}$-B. From this it is also possible to construct the average intercalation voltage, which was computed based on the total energies of the most stable configurations found at each concentration and the energy of lithium in bulk metal ${ }^{[32]}$. Here the voltage at a particular composition is determined by finite differences with respect to the energies of compositions either side of the current value ${ }^{[18]}$. As in previous studies we approximate the free energy by the internal energy and assume that entropic contributions are small at low temperature. The plot of the computed average intercalation voltage corresponding to lithium insertion, computed from energies for compositions in the range 0 to $1 / 2$, is presented in Figure 7.

First of all the preferred location of lithium as a function of increasing content within the $\mathrm{TiO}_{2}$ B structure can be considered. Although the configurational search is not totally exhaustive, leading to some small degree of uncertainty, a clear trend is apparent. The first lithium in the supercell is preferentially positioned at the C site, as previously determined. Similarly, the second lithium adopts the same type of site, but in the other interstitital region between the octahedral layers (there being two conventional cells per supercell in each direction). The next two lithium ions are also able to occupy the more favourable C-type sites, but have to share the same plane as the previously intercalated lithium ions. Consequently, they prefer to reside on the opposite side of the $b$ axis channel and displaced along this direction relative to the first lithium. By the composition of $\mathrm{Li}_{0.125} \mathrm{TiO}_{2}$, a more complex interplay between intrinsic site preference and lithium-lithium Coulombic repulsion begins to occur. For this material, four lithiums are located at the C-type sites, while two are now found at the least favourable A-site, adjacent to the octahedral titania layers. Hence, it is clear that maximising the lithium-lithium distance begins to become sufficiently important that the structure prefers not to populate all C-type sites. At the highest level of lithium incorporation investigated, $\mathrm{Li}_{0.5} \mathrm{TiO}_{2}$, which corresponds to 24 lithium atoms per supercell, the distribution is equally divide between $\mathrm{A}$ and $\mathrm{C}$ sites.

Turning to consider the voltage curve as a function of composition, shown in Figure 7, there are two distinct regions. Initially the voltage exhibits an almost constant value, just in excess of $2.4 \mathrm{~V}$, before decreasing to a value just below $2 \mathrm{~V}$. The second voltage point, corresponding to the average lithium content of 0.0315 , is higher than that of the two neighbouring points, and therefore should disproportionate. However, the voltage difference is only $0.02 \mathrm{eV}$ relative to the previous data point, which is close to the numerical precision, let alone the accuracy of the results. Furthermore, it indicates that investigation of many more intermediate compositions would ideally be required in this region in 
order to obtain more precise averages with respect to lithium composition. Although there is some uncertainity, the observation of an initial higher voltage plateau, followed by a lower voltage region is consistent with the calculated behaviour of other lithium battery materials, including previous first principles studies of rutile and anatase. ${ }^{[22]}$ Indeed, the voltage curve for $\mathrm{TiO}_{2}$ - $\mathrm{B}$ closely resembles that of rutile, as calculated by Koudriachova et al. The decrease in voltage around the lithium fraction of 1/16 corresponds to the point where the population of the less favourable A-sites begins and lithiumlithium interactions become more significant. The initial computed voltage for low concentrations of lithium is comparable to the open circuit voltage of $2.55 \mathrm{~V}$ observed experimentally ${ }^{[5]}$, though as expected the GGA calculation underestimates this quantity.

\section{Conclusions}

In the present study of $\mathrm{TiO}_{2}$-B we have examined the intercalation of lithium over a range of compositions. Towards the dilute limit there are three distinct symmetry unique sites that emerge. The most stable location for the incorporation of lithium in the absence of lithium-lithium interactions is within the interlamellar region between successive sheets of octahedral titania, but closely coordinated by the oxygen atoms that connect the sheets. In contrast, the more favourable pathway for diffusion lies close to the centre of the more open channel structure, parallel to the $b$ axis of the material in accord with experimental observation. In combination, these two results suggest a reason for the initial need to activate the $\mathrm{TiO}_{2}-\mathrm{B}$ in that the lithium has to be promoted from the thermodynamically favoured site to the one in the centre of the channel from where it can rapidly diffuse. This prediction appears to be consistent with present observations regarding the electrochemical behaviour of this polymorph of titania. As the concentration of lithium increases, lithium is forced to populate the least intrinsically stable sites, adjacent to the octahedral layers, in order to mininise the cation-cation interactions. This leads to a drop in the voltage for the material and would lower the mobility of lithium with respect to intercalation. Further work is required to examine the differences in the properties of bulk $\mathrm{TiO}_{2}-\mathrm{B}$, as examined in the present work, and nanostructured form of this material.

\section{Acknowledgements}

We would like to thank iVEC and NCI for the provision of supercomputing facilities. JDG would like to thank the Australian Research Council for funding through a Professorial Research Fellowship. 
Table 1. Energy of lithium incorporation into $\mathrm{TiO}_{2}-\mathrm{B}$ for the three distinct sites, $\mathrm{A}, \mathrm{B}$ and $\mathrm{C}$. Both the absolute internal energy for the process, as well as the relative energy as compared to the most stable site, are given for the stoichiometry of $\mathrm{Li}_{1 / 48} \mathrm{TiO}_{2}-\mathrm{B}$.

\begin{tabular}{|c|c|c|}
\hline Site & $\mathrm{U}_{\text {Intercalation }}(\mathrm{eV})$ & $\Delta \mathrm{U}(\mathrm{kJ} / \mathrm{mol})$ \\
\hline (A) & -3.436 & 32.5 \\
\hline (B) & -3.678 & 9.08 \\
\hline (C) & -3.772 & 0.00 \\
\hline
\end{tabular}


Table 2. Summary of the calculated activation energy barriers $(\Delta \mathrm{U})$ for diffusion along pathways parallel to each crystallographic axis. Here the labels for the sites can be found in Figures 3a-c for the directions parallel to $a, b$, and $c$, respectively, while the arrows indicate the direction of diffusion for which the barrier occurs.

\begin{tabular}{|c|c|c|}
\hline Direction & Sites & $\Delta \mathrm{U}(\mathrm{kJ} / \mathrm{mol})$ \\
\hline$a$ & $\mathrm{~B} \leftarrow \mathrm{C}_{1}$ & 12.2 \\
& $\mathrm{~B} \rightarrow \mathrm{C}_{1}$ & 3.1 \\
\hline$b$ & $\mathrm{C}_{1} \leftrightarrow \mathrm{C}_{2}$ & 96.9 \\
\hline$c$ & $\mathrm{~B}_{1} \leftrightarrow \mathrm{B}_{2}$ & 27.0 \\
\hline & $\mathrm{B} \rightarrow \mathrm{A}_{1}$ & 37.6 \\
& $\mathrm{~B} \leftarrow \mathrm{A}_{1}$ & 14.2 \\
& $\mathrm{~A}_{1} \leftrightarrow \mathrm{A}_{2}$ & 36.4 \\
\hline
\end{tabular}




\section{Figure Captions:}

Figure 1. Bulk $\mathrm{TiO}_{2}-\mathrm{B}$ structure. The unit cell is surrounded by the solid black line, while further images are included to highlight the connectivity across cell boundaries. The structure exhibits open channels perpendicular to the (010) face. Oxygen and titanium atoms are drawn in red and green, respectively.

Figure 2. The predicted lithium ion intercalation sites, $\mathrm{A}, \mathrm{B}$, and $\mathrm{C}$, in the $\mathrm{TiO}_{2}-\mathrm{B}$ lattice for $\mathrm{Li}_{1 / 48} \mathrm{TiO}_{2}-\mathrm{B}$ stoichiometry. The three distinct sites are illustrated in separate figures labeled according to the site type. Here the structure is viewed along the $b$-axis with the direction of the other axes indicated in the figures. Lithium, oxygen and titanium atoms are marked in blue, red and green, respectively. The annotated numbers correspond to the oxygen atoms closest the lithium ion, which is also identifiable through the lack of bonds depicted.

Figure 3. Lithium diffusion pathways within $\mathrm{Li}_{1 / 48} \mathrm{TiO}_{2}-\mathrm{B}$. (a) Two stage diffusion pathway along $a$ direction, involving migration from $\mathrm{B}$ to $\mathrm{C} 1$ and $\mathrm{C} 1$ to $\mathrm{C} 2$. (b) The main diffusion pathway along $b$ direction between two consecutive B sites. (c) Two stage diffusion pathway along $c$ direction, involving B to A1 and A1 to A2. The symmetry related path on the other side of B is also illustrated to indicate the complete diffusion process in the $c$ direction. Lithium, oxygen and titanium atoms are shown in blue, red and green, respectively.

Figure 4. Potential energy surfaces for lithium migration through $\mathrm{Li}_{1 / 48} \mathrm{TiO}_{2}-\mathrm{B}$ leading to diffusion parallel to the crystallographic $a$ axis. (a) The energies for diffusion along the $\mathrm{C} \rightarrow \mathrm{B}$ pathway . (b) The energies for diffusion along the $\mathrm{C} 1 \rightarrow \mathrm{C} 2$ pathway. Energy surfaces are obtained relative to the minimum energy configuration.

Figure 5. Potential energy surfaces for lithium migration through $\mathrm{Li}_{1 / 48} \mathrm{TiO}_{2}-\mathrm{B}$ leading to diffusion parallel to the crystallographic $b$ axis. It shows the computed energies for diffusion along the B1 $\rightarrow$ B2 pathway (squares). An equivalent energy surface for B1 $\leftarrow$ B2 pathway (triangles) is also presented.

Figure 6. Potential energy surfaces for lithium migration through $\mathrm{Li}_{1 / 48} \mathrm{TiO}_{2}-\mathrm{B}$ leading to diffusion parallel to the crystallographic $c$ axis. (a) The computed relative energy for diffusion along the B $\rightarrow$ A1 pathway. (b) The computed relative energy for diffusion along the A1 $\rightarrow$ A2 pathway.

Figure 7. The average intercalation voltage curve for $\mathrm{Li}_{\mathrm{x}} \mathrm{TiO}_{2}-\mathrm{B}$ computed based on intercalation energies for lithium concentrations between $x=0$ and $1 / 2$. Crosses represent the data points, while the line is included only as a guide. 


\section{References}

[1] A. R. Armstrong, G. Armstrong, J. Canales and P. G. Bruce, Angew. Chem. Int. Ed. 2004, 43, 22862288.

[2] G. Armstrong, A. R. Armstrong, J. Canales and P. G. Bruce, Chem. Commun. 2005, 2454.

[3] A. R. Armstrong, G. Armstrong, J. Canales and P. G. Bruce, J. Power Sources 2005, 146, 501-506.

[4] G. Armstrong, A. R. Armstrong, P. G. Bruce, P. Reale and B. Scrosati, Adv. Mater. 2006, 18, 25972600.

[5] G. Armstrong, A. R. Armstrong, J. Canales and P. G. Bruce, Electrochemical and Solid-State Letters 2006, 9, A139-A143.

[6] A. R. Armstrong, G. Armstrong, J. Canales, R. Garcia and P. G. Bruce, Adv. Mater. 2005, 17, 862 865.

[7] T. P. Feist and P. K. Davies, J. Solid State Chem. 1992, 101, 275-295.

[8] G. Nuspl, K. Yoshizawab and T. Yamabe, J. Mater. Chem. 1997, 7, 2529 - 2536.

[9] M. Tournoux, R. Marchand and L. Brohan, Prog. Solid State Chem. 1986, 17, 33-52.

[10] R. Marchand, L. Brohan and M. Tournoux, Mater. Res. Bull. 1980, 15, 1129-1133.

[11] M. Wagemaker, A. Van Der Ven, D. Morgan, G. Ceder, F. M. Mulder and G. J. Kearley, Chemical Physics 2005, 317, 130-136.

[12] G. Ceder, Y. M. Chiang, D. R. Sadoway, M. K. Aydinol, Y. I. Jang and B. Huang, Nature 1998, 392, 694-696.

[13] G. Ceder, Science 1998, 280, 1099-1100.

[14] K. Kang, Y. S. Meng, J. Breger, C. P. Grey and G. Ceder, Science 2006, 311, 977-980.

[15] A. Van der Ven and G. Ceder, Electrochemical and Solid-State Letters 2000, 3, 301-304.

[16] A. Van der Ven and G. Ceder, J. Power Sources 2001, 97-98, 529-531.

[17] G. Ceder, A. Van der Ven and M. Aydinol, JOM 1998, 50, 35-40.

[18] J. S. Braithwaite, C. R. A. Catlow, J. D. Gale and J. H. Harding, Chem. Mater. 1999, 11, 19901998.

[19] M. M. Islam, T. Bredow, S. Indris and P. Heitjans, Phys. Rev. Lett. 2007, 99, 145502-145504.

[20] M. V. Koudriachova, N. M. Harrison and S. W. de Leeuw, Phys. Rev. B 2002, 65, 1-12.

[21] M. V. Koudriachova, N. M. Harrison and S. W. de Leeuw, Solid State Ionics 2003, 157, 35-38.

[22] M. V. Koudriachova, N. M. Harrison and S. W. de Leeuw, Phys. Rev. Lett. 2001, 86, 1275 -1278.

[23] R. Hoffmann, J. Chem. Phys. 1963, 39, 1397-1412.

[24] T. Hughbanks and R. Hoffmann, J. Am. Chem. Soc. 1983, 105, 3528-3537.

[25] M. Koudriachova, S. W. de Leeuw and N. M. Harrison, Chem. Phys. Lett. 2003, 371, 150-156.

[26] M. V. Koudriachova and S. W. de Leeuw, Phys. Rev. B 2004, 69, 054106.

[27] M. V. Koudriachova, N. M. Harrison and S. W. de Leeuw, Computational Materials Science 2002, 24, 235-240.

[28] J.M. Soler, E. Artacho, J. D. Gale, A. García, J. Junquera, P. Ordejón, and D. Sánchez-Portal, J. Phys. Cond. Matter, 2002, 14, 2745-2779. 
[29] N. Troullier and J. L. Martins, Phys. Rev. B 1991, 43, 1993-2006.

[30] J. Junquera, Ó. Paz, D. Sánchez-Portal and E. Artacho, Phys. Rev. B 2001, 64, 235111.

[31] D. Vanderbilt, Phys. Rev. B 1990, 41, 7892-7895.

[32] M. K. Aydinol, A. F. Kohan, G. Ceder, K. Cho and J. Joannopoulos, Phys. Rev. B 1997, 56, 1-12.

[33] L. Brohan and R. Marchand, Solid State Ionics 1983, 9-10, 419-424.

[34] M. V. Koudriachova, N. M. Harrison and S. W. de Leeuw, Solid State Ionics 2004, 175, 829-834.

[35] J. Muscat, V. Swamy and N. M. Harrison, Phys. Rev. B 2002, 65, 1-15.

[36] L. D. Noailles, S. D. Johnson, J. T. Vaughey and M. M. Thackeray, J. Power Sources 1999, 81-82, 259-263.

[37] M. R. Ranade, A. Navrotsky, H. Z. Zhang, J. F. Banfield, S. H. Elder, A. Zaban, P. H. Borse, S. K. Kulkarni, G. S. Doran and H. J. Whitfield, Proc. Natl. Acad. Sci. 2002, 99, 6476-6481.

[38] A. Stashans, S. Lunell, R. Bergström, A. Hagfeldt and S.-E. Lindquist, Phys. Rev. B 1996, 53, 159-170.

[39] L. R. Wallenberg, M. Sanati and A. Andersson, Microsc. Microanal. Microstruct. 1990, 1, 357364.

[40] B. Zachau-Christiansen, K. West, T. Jacobsen and S. Atlung, Solid State Ionics 1988, 28, 11761182.

[41] J. P. Perdew, K. Burke and M. Ernzerhof, Phys. Rev. Lett. 1996, 77, 3865-3868.

[42] F. Zhou, M. Cococcioni, C.A. Marianetti, D. Morgan and G. Ceder, Phys. Rev. B 2004, 70, 235121.

[43] I. Leonov, A.N. Yaresko, V.N. Antonov, U. Schwingenschlögl, V. Eyert and V.I. Anisimov, J. Phys.: Condens. Matter 2006, 18, 10955-10964.

[44] J. Moreno and J. M. Soler, Phys. Rev. B 1992, 45, 13891-13898.

[45] S.D. Fleming and A.L. Rohl, Z. Krist., 2005, 220, 580-584.

[46] R. C. Hoft, J. D. Gale and M. J. Ford, Mol. Simul. 2006, 32, 595 - 600.

[47] V. L. Vinograd, B. Winkler, A. Putnis, J. D. Gale and M. H. F. Sluiter, Chem. Geol. 2006, 225, 304-313.

[48] M. Zukalova, M. Kalbac, L. Kavan, I. Exnar and M. Graetzel, Chem. Mater. 2005, 17, 1248-1255.

[49] R. K. B. Gover, J. R. Tolchard, H. Tukamoto, T. Murai and J. T. S. Irvine, Journal of the Electrochemical Society 1999, 146, 4348-4353.

[50] R. Hengerer, L. Kavan, P. Krtil and M. Gratzel, J. Electrochem. Soc. 2000, 147, 1467-1472. 